\title{
EchoGéo
}

\section{Risques de gel accru liés à la présence d'ouvrages linéaires en remblai}

Le cas des lignes nouvelles du TGV Méditerranée et de la future ligne du TGV Est européen

Hervé Quenol, Gérard Beltrando et Sébastien Bridier

\section{CpenEdition}

Journals

Édition électronique

URL : https://journals.openedition.org/echogeo/1052

DOI : 10.4000/echogeo.1052

ISSN : 1963-1197

Éditeur

Pôle de recherche pour l'organisation et la diffusion de l'information géographique (CNRS UMR 8586)

Référence électronique

Hervé Quenol, Gérard Beltrando et Sébastien Bridier, « Risques de gel accru liés à la présence d'ouvrages linéaires en remblai », EchoGéo [En ligne], 1 | 2007, mis en ligne le 22 février 2008, consulté le 01 août 2021. URL : http://journals.openedition.org/echogeo/1052 ; DOI : https://doi.org/10.4000/ echogeo.1052

Ce document a été généré automatiquement le 1 août 2021.

EchoGéo est mis à disposition selon les termes de la licence Creative Commons Attribution - Pas d'Utilisation Commerciale - Pas de Modification 4.0 International (CC BY-NC-ND) 


\title{
Risques de gel accru liés à la présence d'ouvrages linéaires en remblai
}

Le cas des lignes nouvelles du TGV Méditerranée et de la future ligne du TGV Est européen

\author{
Hervé Quenol, Gérard Beltrando et Sébastien Bridier
}

Introduction

1 Depuis quelques années, face aux inquiétudes liées aux catastrophes climatiques ou météorologiques, à la dégradation de la qualité de l'air ou à la question du possible réchauffement de la planète, certains géographes ont fait de l'action anthropique sur le climat un de leurs pôles de recherche.

2 Parmi ces thèmes de recherche il en est un, relativement nouveau, au moins en France, qui a été peu abordé par les scientifiques : celui de l'impact des grandes infrastructures linéaires de transport (remblais ferroviaires ou routiers) sur la circulation de l'air froid en surface et donc finalement sur la production agricole. Pourtant, en modifiant la nature et la rugosité de la surface, un ouvrage d'art, de plusieurs mètres de haut et de plusieurs centaines de mètres de long, a une influence sur le système climatique local et lorsque des cultures (principalement la viticulture et l'arboriculture) sont sensibles à certains aléas climatiques (gel, vent), l'introduction d'un nouvel obstacle dans le milieu peut permettre ou accentuer les dommages aux plantes. Jusqu'à ces dernières années, bien que souvent observé par les agriculteurs, ce type d'impact a peu interpellé les scientifiques ; probablement, parce que l'aire d'influence de ces obstacles est très localisée et qu'il paraissait difficile de le prouver par manque de référence à la situation avant la présence du remblai. Mais aussi parce que ce risque supplémentaire, provoqué par ces variations météorologiques et climatiques locales, est d'ordre économique et ne concerne qu'une minorité ou une catégorie spécifique d'agriculteurs (par exemple des viticulteurs).

3 L'objectif de ce type d'étude vise donc à évaluer le différentiel thermique créé par l'ouvrage en remblai et à le cartographier de manière à fournir un document facilitant 
les négociations entre le Maître d'ouvrage et la profession agricole (agriculteurs, syndicat professionnel, chambre d'agriculture) dans une optique de gestion de ce risque supplémentaire (le bas des coteaux est souvent un espace relativement gélif, l'ouvrage de transport ne fait qu'accentuer ce risque). Lorsqu'il y a un enjeu économique avéré, ce type de démarche commence à être intégré dans les enquêtes d'utilité publique avant la construction de l'ouvrage linéaire en remblai. Cette étude permet de réduire les conflits potentiels entre les professionnels agricoles et le Maître d'ouvrage : soit en validant le tracé si le risque supplémentaire parait minime, soit en apportant des modifications à l'ouvrage (ouverture dans le remblai, changement de la forme et/ou de la hauteur...) ou encore en négociant des compensations financières pour l'agriculteur dans le cas où le risque supplémentaire parait évident et les possibilités d'aménagements insuffisantes.

1. Une prise en compte récente en France: l'étude pionnière sur la future Ligne à Grande Vitesse du TGV Est européen

4 Les premières études sur l'influence climatique des remblais ont été réalisées en Allemagne. En 1980, Geiger a montré que la présence d'obstacles de plusieurs mètres de haut accentuait le risque de gel dommageable pour les cultures viticoles dans la Haardt (Allemagne). En conclusion de son article, Geiger préconisait que l'impact climatique soit intégré dans les projets de grandes constructions : "les études topoclimatiques devraient être prises en considération pour la construction des routes... on a dressé récemment des remblais routiers jusqu'à une hauteur de $10 \mathrm{~m}$ au-dessus des fonds de vallée. Ceux-ci peuvent provoquer des variations climatiques locales, qui ont des conséquences négatives sur la viticulture ou sur l'habitat ".

5 En France, au début des années 1990, la question de l'impact climatique du remblai ferroviaire sur le risque gélif a été posée lors de la présentation de l'avant-projet sommaire (APS) de la Ligne à Grande Vitesse (LGV) Est européenne. En réponse aux inquiétudes des professionnels viticoles champenois, sur la probabilité d'une accentuation du risque gélif printanier après l'implantation du remblai de la LGV, la SNCF puis Réseau Ferré de France (RFF, a été créé en 1997) ont timidement financées une première étude confiée à des universitaires afin d'essayer d'évaluer ce "risque climatique » (Beltrando et al., 1995). Une expérimentation agroclimatique réalisée dans le vignoble champenois sur les communes de Verzenay et Sillery (sud-est de Reims) et basée sur des mesures réalisées à proximité d'une bâche simulant le futur remblai, a permis d'observer qu'un remblai, aménagé dans le bas d'un coteau sur une longueur de $780 \mathrm{~m}$. et avec une hauteur atteignant $5 \mathrm{~m}$, accentuait, pour les situations météorologiques de type radiatives (ciel clair vent faible ou nul), le refroidissement nocturne en bloquant les écoulements gravitaires et en créant un lac d'air froid (figure 1). 


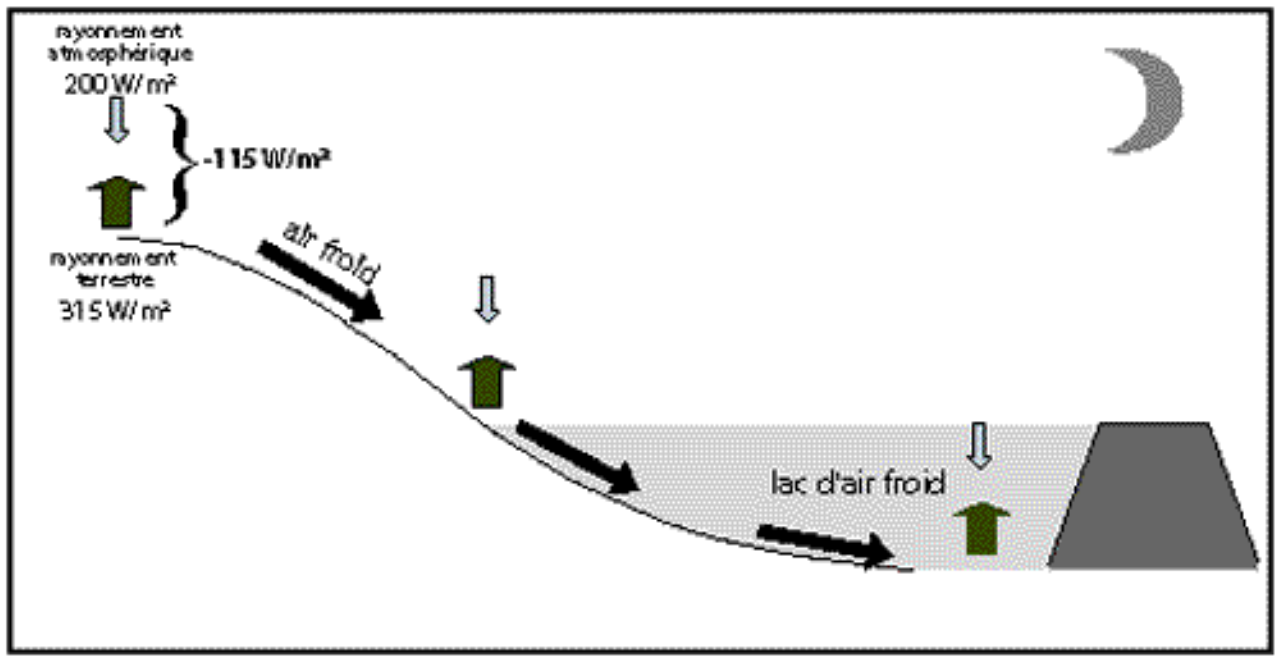

Figure 1 - Formations des écoulements gravitaires et du « lac d'air froid » en amont du remblai (valeurs moyennes de rayonnement pour la Champagne en avril).

$6 \mathrm{Au}$ printemps, à partir du débourrement (moyenne mi avril en Champagne), les bourgeons des vignes sont sensibles aux basses températures: à partir de $-2^{\circ} \mathrm{C}$, ils peuvent geler et compromettre ainsi une partie de la récolte. La présence d'un nouvel obstacle représente donc, localement, un risque supplémentaire de gel notamment pour les situations radiatives où le fort déficit énergétique au niveau du sol favorise le refroidissement nocturne (7 et al., 1995; Bridier et al., 1995). Réseau Ferré de France (RFF), par l'intermédiaire du Maître d'œuvre du tronçon traversant la Montagne de Reims (société Tractebel Coyne et Bellier), a proposé une modification des caractéristiques du tracé dans le secteur afin de réduire le volume du lac d'air froid abaissement du profil de la ligne, diminution de la longueur du remblai, remodelage du terrain en amont par l'apport de $60000 \mathrm{~m}^{3}$ de terres, mise en place d'un rayon de courbure exceptionnel dans ce secteur (figure 2). 
Figure 2 - Modification du tracé proposée par le Maître d'œuvre et le Maître d'ouvrage dans le secteur des Fonds de Sillery

\section{Fonds de Sillery - Coupe transversale

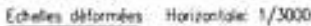 Vericale $1 / 100$}

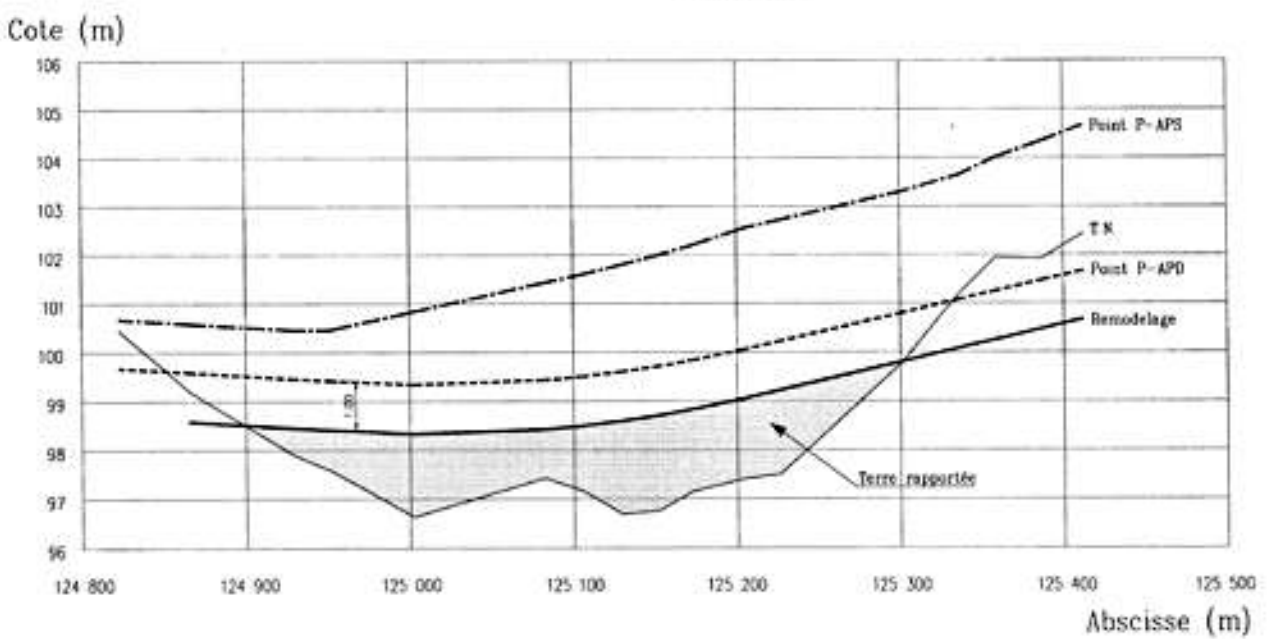

$(\mathrm{APS}=$ Avant projet sommaire $; \mathrm{APD}=$ Avant - projet définitif $; \mathrm{TN}=$ terrain naturel $)$

7 Dans le cadre de l'avant projet définitif (APD), une seconde étude s'est déroulée au printemps 2001 pour estimer, toujours avant construction, l'impact du nouvel aménagement; et devant l'impact médiatique de cette première étude, l'utilité d'intégrer l'aspect climatique local dans les études d'impact a émergé dans d'autres tronçons de la LGV-Est ${ }^{1}$ (Vrigny et Les Mesneux au sud-ouest de Reims) ainsi que sur la LGV Méditerranée (Valence à Marseille²).

Ces premières études d'impact issues d'une demande émanant de la profession agricole (syndicats viticoles, chambres d'agriculture) se sont multipliées ces dernières années. Ainsi, en 2002, la Direction Départementale de l'Equipement du Bas-Rhin, a intégré ce type de démarche dans une enquête d'utilité publique relative au projet de construction de la déviation de la RN59 qui doit traverser le vignoble alsacien au niveau de la commune de Châtenois ${ }^{3}$ (Beltrando et al, 2003). Sur ce site, la DDE a demandé une étude d'impact climatique du remblai de la route nouvelle, afin de proposer des aménagements pour limiter ce risque (en particulier la création d'ouvertures dans le remblai pour favoriser l'écoulement de l'air froid). Mais, devant les résultats de l'étude et devant la crainte des viticulteurs de trouver des traces de polluants, le tribunal administratif de Strasbourg a annulé ce projet de déviation.

de ces différentes études, des techniques de mesures météorologiques et de modélisation spatiale adaptées aux échelles fines ont été imaginées et testées afin évaluer l'impact agro-climatique des remblais et de fournir un document cartographique exploitable par la profession agricole et le Maitre d'ouvrage.

2. Méthodologie : mesures et modélisation spatiale adaptées aux échelles fines

10 Aux échelles spatiales fines, le climat de la couche limite est fortement tributaire des conditions de surface. La morphologie, les aspérités et la nature de la surface (relief, végétation, obstacles anthropiques, ...) modifient le comportement des variables météorologiques et engendrent ainsi une forte variabilité spatiale de ces phénomènes sur des espaces relativement restreints (Yoshino, 1975; Mahrt, 1986; Oke, 1987; 
Carrega, 1994). Pour évaluer l'impact d'un élément perturbateur supplémentaire comme un remblai, la méthode de mesures et de modélisation spatiale doit prendre en compte les caractéristiques de la surface (topographie, occupation du sol, ...).

\subsection{Mesures sur le terrain : fixes et itinérantes}

11 L'observation du climat aux échelles fines implique une méthode d'acquisition des données météorologiques adaptée afin de déterminer l'influence du milieu sur les variables météorologiques. L'emplacement des postes de mesures ne peut donc pas être défini en fonction des normes standard ${ }^{4}$ mais en fonction de l'influence des différents éléments de la surface (dont le remblai) qui sont susceptibles de modifier la météorologie locale. La densité du réseau météorologique conventionnel est trop lâche pour mettre en évidence cette variabilité spatiale du climat. L'observation des paramètres météorologiques aux échelles fines passe donc par la mise en place d'un réseau de mesures temporaires adapté à la configuration du milieu et à l'objectif de l'étude.

L'observation de la variabilité des paramètres météorologiques aux échelles fines nécessite l'installation de postes de mesures répartis sur l'ensemble du terrain d'expérimentation. Les données sont obtenues soit de manière ponctuelle par l'intermédiaire de mesures itinérantes effectuées par un ou plusieurs opérateurs, soit simultanément sur plusieurs points grâce à des capteurs reliés en réseau. Dans le premier cas, le matériel peut être meilleur marché, mais il faut une présence humaine permanente lors des nuits froides et le décalage temporel entre chaque relevé constitue un inconvénient pour les comparaisons. La seconde technique de mesures présente l'intérêt de pallier le problème d'intervalle entre les relevés ; cependant, le coût des capteurs peut devenir une contrainte.

Dans le cadre des études d'impact climatique avant la construction de la ligne ferroviaire, où l'objectif principal est d'évaluer le risque supplémentaire probable afin d'apporter des modifications au tracé, l'expérimentation sur le terrain s'est déroulée de deux manières :

La simulation de l'obstacle, que constitue le remblai, est réalisée par l'intermédiaire d'une bâche en plastique dont la hauteur et la longueur sont similaires à celles du remblai (photo 1). Les mesures des variables atmosphériques sont réalisées sur le terrain initial sans, puis avec, la bâche simulant le remblai, de manière à obtenir un différentiel entre les deux situations. C'est ce type de méthode qui a été entreprise lors de l'étude d'impact du remblai de la LGV-Est sur le gel printanier dans le vignoble de Verzenay/Sillery.

Vues de la bâche simulant le futur remblai sur le site de Verzenay/Sillery en 1994 et en 2001. (Cliché : H. Quénol)

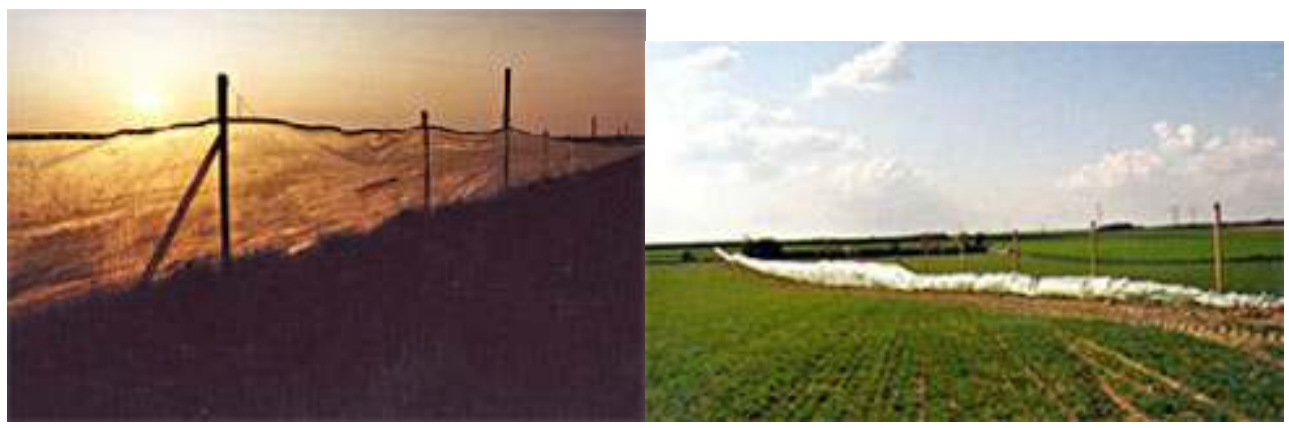


La simulation du remblai étant relativement onéreuse à mettre en place, la seconde méthode expérimentale consiste à effectuer des mesures des variables atmosphériques (par exemple, les températures et les écoulements nocturnes) sur le site avant l'implantation de l'obstacle ferroviaire. L'observation du climat local permet ensuite d'évaluer et de simuler par l'informatique l'impact du futur remblai. Par exemple, pour déterminer l'influence du remblai de la LGV-Est sur le risque gélif dans le vignoble de Vrigny (Sud-ouest de Reims), une première campagne terrain (2002) a permis d'évaluer la répartition spatiale des températures minimales et la localisation du lac d'air froid sur le site initial avant l'implantation de l'obstacle ferroviaire. Ensuite, l'impact du remblai a été simulé dans un Système d'Information Géographique de manière à évaluer l'extension du lac d'air froid (cf. méthodologie développée dans la partie 3.2). Une seconde série de mesures sur le terrain (2005) effectuée après la construction du remblai a montré que l'obstacle accentuait le blocage de l'air froid et a permis de valider la simulation informatique. La figure 3 montre que les températures minimales les plus froides ont été relevées dans les secteurs où la simulation informatique prévoit une accentuation du risque gélif.

Figure 3 - Simulation du risque gélif par SIG (2002) et températures minimales de la nuit du 21 au 22 avril 2005 (après l'implantation du remblai ferroviaire).

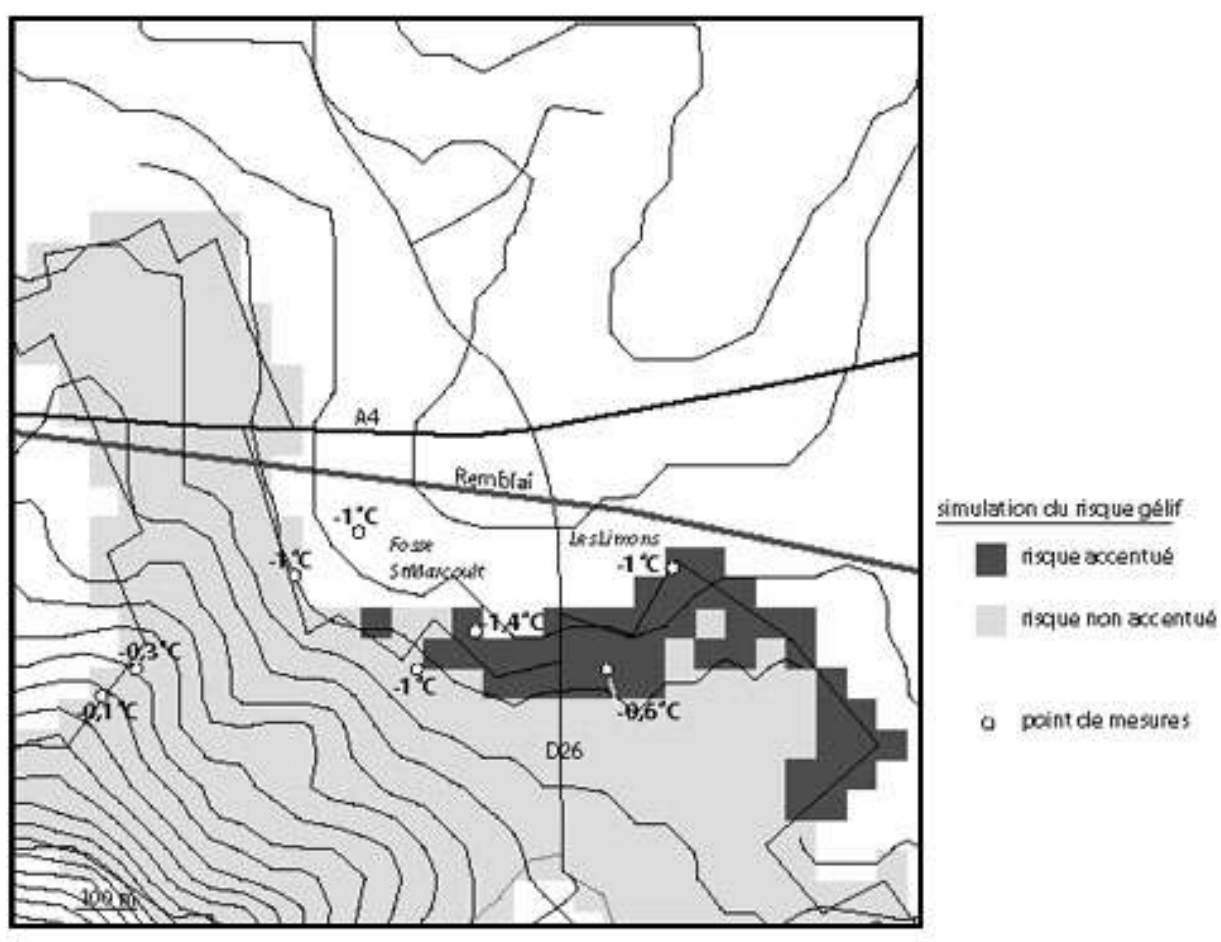

Concrètement, les mesures in situ destinées à étudier la variabilité spatiale du gel printanier nocturne se déroulent en plusieurs étapes :

- Une première série de mesures itinérantes avec une girouette ultra légère ${ }^{5}$ permet de déterminer l'aérologie locale nocturne en situation radiative. En effet, l'air froid issu des déperditions énergétiques du sol s'écoule par gravité suivant les lignes de pente.

- Une fois repérés les différents couloirs dans lesquels l'air froid va s'écouler (intensité du flux et fonction du bassin versant en amont du point de mesures), des capteurs thermiques sont disposés en fonction des caractéristiques du site (pente, cuvette, proximité du remblai...) et 
de manière à quadriller au mieux l'espace étudié et fonction des moyens financiers et humains disponibles). Ces capteurs vont enregistrer la température de l'air, hors abri météorologique (dans le jargon des météorologues et climatologues cela est appelé « température en indice actinothermique »), suivant un pas de temps défini et simultanément sur les différents postes de mesures. Ces mesures vont permettre d'évaluer et de quantifier les secteurs les plus froids pour un type de situation météorologique donnée.

- Après avoir acquis les données sur le terrain par l'intermédiaire de mesures météorologiques, l'étape suivante consiste à réaliser une spatialisation sur l'ensemble du site afin d'obtenir un document cartographique représentant de façon exhaustive les différents secteurs où le risque agro climatique est accentué par l'ouvrage en remblai. Le passage du « terrain » à la spatialisation du phénomène nécessite l'utilisation d'un outil s'inscrivant dans la démarche scientifique définit au cours des mesures météorologiques, c'est-à-dire, étudier l'influence des différents éléments du milieu (topographie, occupation du sol, remblai...) pouvant modifier le comportement des variables atmosphériques.

2.2. Spatialisation du risque accru : vers une modélisation informatique par Système d'Information Géographique

Après avoir acquis les données sur le terrain, une modélisation spatiale sur l'ensemble de l'espace étudié permet de représenter les aires concernées par un risque supplémentaire et de produire des documents cartographiques destinés aux décideurs.

En situation radiative, la variabilité climatique locale est en grande partie provoquée par les effets locaux de la topographie et de la nature de la surface. La principale difficulté de la modélisation spatiale aux échelles fines réside dans la manière d'intégrer l'influence de ces paramètres locaux sachant qu'ils agissent sur les variables météorologiques à différentes échelles spatiales et temporelles. Pour y parvenir, il faut utiliser une méthode de cartographie permettant d'établir les relations entre les caractéristiques de surface (morphologie, occupation du sol) et les variables météorologiques. Comme l'indique Kergomard et al. (2002) «Les méthodes les plus prometteuses de cartographie à échelle fine (...) reposent sur une interpolation supervisée des mesures localisées, utilisant des relations statistiques (généralement des régressions multiples) entre les paramètres climatologiques mesurés et des descripteurs quantifiés » de l'état de la surface. L'utilisation du Système d'Information Géographique (SIG) est un moyen approprié pour ce type de modélisation spatiale. Le SIG permet de mettre en relation les données mesurées ponctuellement sur le site d'étude (température, vitesse et direction des brises thermiques...) avec les différents obstacles (relief, remblai...) qui influent sur la variabilité spatiale du phénomène climatique.

Les méthodes de spatialisation mises en place ont évolué au fil des études réalisées.

- En 1994, lors de la première phase de l'étude d'impact du remblai du futur TGV Est européen dans le vignoble de Verzenay/Sillery, la cartographie du risque gélif a été réalisée manuellement après l'observation des feuilles de la vigne après des épisodes gélifs. En effet, en automne, lors des premières gelées (dont les conditions atmosphériques sont similaires aux situations printanières), les feuilles des vignes, situées dans les secteurs les plus froids, jaunissent puis rougissent. Même si ce type de carte parait rudimentaire, elle s'est avérée relativement efficace pour spatialiser, en automne, les limites des premières gelées d'origine radiatives, mais elle demande une bonne connaissance des plantes car l'âge de la vigne peut aussi avoir des conséquences sur la couleur des feuilles qui subissent le gel. 
21 - L'utilisation du SIG permet de confronter sous la forme de couches de données, les paramètres atmosphériques avec les caractéristiques de l'espace (topographie, remblai...) susceptibles de modifier le climat local et la vulnérabilité du végétal (variété, niveau de croissance...) (Bridier et al., 2004). La variabilité spatiale du gel printanier étant tributaire de la topographie (l'air froid s'écoule suivant les lignes de pente) et des différents obstacles pouvant ralentir voir bloquer l'air froid (cuvette, remblai), la superposition des couches représentant la pente et la direction théorique des écoulements gravitaires (réalisés à partir d'un Modèle Numérique de Terrain), la position et la hauteur du remblai ainsi que la localisation des cultures sensibles, permettent de spatialiser le risque de gel (encadré).

Par exemple, dans le cadre de l'étude d'impact du remblai de la LGV-Méditerranée sur le gel printanier, une estimation de la surface du "lac d'air froid" a été réalisée en considérant que l'obstacle aura une hauteur de $10 \mathrm{~m}$ de haut dans le fond du secteur, qu'il est horizontal et que les écoulements gravitaires s'écoulent en direction de la ligne ferroviaire. La combinaison entre la couche comportant la hauteur du remblai et l'altimétrie a permis d'évaluer l'air d'extension du lac d'air froid. En croisant cette donnée avec celle des cultures vulnérables au gel, nous obtenons pour une surface totale de 120 hectares où l'aléa gel est accentué par la présence de l'obstacle ferroviaire, une surface d'environ 45 hectares de parcelles fruitières qui pourraient être soumises à un risque supplémentaire de gel printanier après la construction de la LGVMéditérranée.

Etapes de la spatialisation du risque gélif dans un SIG

L'utilisation du SIG est un moyen approprié pour ce type de modélisation spatiale puisqu'il permet de mettre en relation les différentes couches d'informations. Certaines informations nécessitent des traitements de données préalables (interpolation de champs d'altitude, calcul de pentes, ...), le SIG se compose de plusieurs logiciels (Mapinfo et Surfer) et d'une passerelle informatique qui assure une liaison entre ces logiciels qui ont des fonctions spécifiques. Cette passerelle est un ensemble d'objets vectoriels carrés qui forment une grille continue dans l'espace de travail. A chaque carré de la grille peuvent être associés plusieurs attributs. Par exemple, les informations liées à l'altitude où à la pente, dissociées lors des différents traitements, deviennent les attributs d'un même objet (Bridier et al., 2004). Après divers traitements informatiques et statistiques, l'ensemble des informations est intégré dans le logiciel vectoriel (Mapinfo) sous la forme de couches qui, une fois superposées, permettent d'obtenir une représentation spatiale des phénomènes météorologiques étudiés et une cartographie exhaustive des secteurs où le risque gélif est accru par l'ouvrage ferroviaire. Les cartes finales sont en mode vectoriel afin d'obtenir la précision nécessaire à ce niveau d'échelle.

Après avoir intégré dans le SIG les caractéristiques du remblai et des murs antibruit (hauteur, présence d'ouvertures, ...), les données liées à la morphométrie (altitude, pente, direction théorique des écoulements gravitaires, ...) ont été calculées et intégrées dans Mapinfo par l'intermédiaire de la grille (passerelle informatique) composée ici de carrés de $25 \mathrm{~m}$ de côté. Connaissant la topographie de l'espace étudié ainsi que la hauteur de l'obstacle ferroviaire, nous pouvons estimer la surface du « lac d'air froid " et donc estimer la surface couverte par de l'air froid lié à la présence du remblai. Ensuite, ces résultats sont combinés avec les parcelles fruitières les plus sensibles au refroidissement nocturne printanier ou avec le vignoble (vulnérabilité du végétal) afin d'obtenir une cartographie de la surface arboricole où le risque gélif est accentué par le remblai. 
Synthèse, conclusion probablement de nombreuses avancées méthodologiques dans les années à venir facilitent l'intégration locale de grands ouvrages qui aujourd'hui ne peuvent plus être imposés aux populations riveraines sans négociations. Elles permettent aussi d'améliorer l'image de marque des grandes entreprises publiques (RFF, DDE...) qui montrent ainsi leur capacité à prendre de nombreuses précautions et éventuellement à mettre en place des aménagements spécifiques dans leurs ouvrages avant qu'ils ne traversent des espaces agricoles à forte valeur ajoutée (création d'ouverture dans le remblai, modification de la hauteur lorsque cela est techniquement possible...).

Remerciements :

Ces études ont été financées par Réseau Ferré de France (LGV est européenne et LGV méditerranée). En Champagne, elles ont aussi reçu un soutien matériel ou technique du Comité Interprofessionnel du Vin de Champagne, du Syndicat Général des Vignerons, du bureau d'étude Tractebel Coyne Bellier et Ingérop . En Provence, elle ont été soutenues par les techniciens de la Chambre d'Agriculture des Bouches du Rhône ainsi que par ceux de la station d'expérimentation arboricole «La Pugère » à Mallemort.

\section{BIBLIOGRAPHIE}

Beltrando G., Bridier S., Madelin M. Quénol H., 2002. Evaluation de l'impact du remblai de la ligne TGV Est sur les écoulements gravitaires et les gelées de printemps dans le vignoble de Champagne. Rapport d'étude pour Tractebel, Réseau Ferré de France (RFF) et le Syndicat Général des Vignerons de la Champagne, $30 \mathrm{p}$.

Beltrando G., Bridier S., Quénol H., 1995. Estimation des modifications générées par la construction de la future ligne du TGV Est Européen dans le vignoble champenois. Rapport d'étude pour le Syndicat Général des Vignerons de la Champagne, $56 \mathrm{p}$. 
Beltrando G., Quénol H. et Bridier S., 2002. Evaluation de l'impact du futur remblai de la ligne à Grande Vitesse Est Européenne sur les gelées de printemps dans les vignobles de Vrigny et des Mesneux (Champagne). Rapport d'étude pour le Groupement ISL, Réseau Ferré de France (RFF) et le Syndicat Général des Vignerons de la Champagne, $24 \mathrm{p}$.

Bridier S., Quénol H., Beltrando G., 1995. Evaluation de l'influence d'un ouvrage linéaire en remblai - le TGV Est - sur l'écoulement de l'air froid. Publications de l'Association Internationale de Climatologie, vol. 8, p. 233-240.

Bridier S., Quénol H. et Beltrando G., 2004. Simulation du potentiel de refroidissement en situation radiative : application aux terroirs des Fonds de Sillery dans le vignoble de Champagne. Revue Internationale de Géomatique. 14, p. 119-132.

Carrega P., 1994. Topoclimatologie et habitat. Revue d'Analyse Spatiale Quantitative et Appliquée, Th. Et., 35 \& 36, 408 p.

Geiger M., 1980. Recherches topoclimatiques au Palatinat. Recherches Géographiques à Strasbourg, $\mathrm{n}^{\circ} 13-14$, p. 95-102.

Kergomard C., Bigot S., Charabi Y., Leriche P., 2002. Climat urbain et qualité de l'air : approches géographiques et collaborations pluridisciplinaires. Bulletin Association de Géographes Français, 4, p. 462-473.

Mahrt L., 1986. Nocturnal topoclimatology. WMO, 117, TD 132, Genève, Suisse, 76 p.

Oke T.R., 1987. Boundary Layer Climates. Second Edition, London, 372 p.

Quénol H., 2002. Climatologie appliquée aux échelles spatiales fines : influence des haies brise-vent et d'un remblai ferroviaire sur le gel printanier et l'écoulement du mistral. Thèse de l'USTL, Edition ANRT, ISBN 2-284-04081-0, $283 \mathrm{p}$.

Yoshino M., 1975. Climate in a small area: an introduction to local meteorology. University of Tokyo Press, 549 p.

\section{NOTES}

1. En 2002, l'étude d'impact de la LGV-Est dans les vignobles de Vrigny et des Mesneux a mis en évidence un risque accru de gel. RFF et les bureaux d'études concernés ont modifié le profil du remblai. Des ouvertures dans le remblai et dans les murs antibruit ont été réalisées afin de favoriser l'écoulement de l'air froid et de limiter son accumulation (Beltrando et al, 2002).

2. Suite aux inquiétudes des arboriculteurs de la commune de Mallemort (Bouches du Rhône), une étude d'impact climatique de la LGV-Méditerranée a montré que l'obstacle ferroviaire accentuait le risque de gel printanier et modifiait l'écoulement du mistral, ce qui engendrait des problèmes de qualité pour les fruits (coups et frottements sur les fruits) (Quénol, 2002).

3. En 2002, une étude de terrain et une simulation informatique ont montré que le risque gélif serait accentué après la construction du remblai (Beltrando et al, 2002). Le tribunal administratif a annulé le projet de construction de la déviation en demandant à la DDE d'apporter des modifications. En 2005, une deuxième étude (toujours en cours) a pour objectif d'évaluer l'impact de ce nouveau projet (Beltrando et al, 2006).

4. Selon l'OMM (1990), « La station météorologique doit être située en terrain dégagé, éloignée des bâtiments et des rideaux d'arbres de plus de 10 fois leur hauteur ». 
5. Girouette ultra légère en balsa (bois exotique) conçue spécifiquement à la mesure d'écoulements de l'air inférieurs à $1 \mathrm{~m} / \mathrm{s}$ et indétectables avec les girouettes classiques (conception Quénol et Bridier).

\section{RÉSUMÉS}

Sur un coteau, le remblai d'un ouvrage de transport, en créant un obstacle à l'écoulement naturel de l'air froid superficiel, favorise la formation d'un lac d'air froid. Au printemps, cet obstacle peut accroître le risque de gel dommageable pour les cultures qui sont vulnérables à cet aléa (vigne, arbres fruitiers...). A partir de mesures météorologiques aux échelles fines, réalisées lors des nuits froides de type "radiatives" (vent faible et ciel clair) et en simulant le remblai sur une longueur conséquente par l'intermédiaire d'un bâche, il est possible d'évaluer grossièrement le différentiel thermique que va constituer cet ouvrage. Une autre méthode, basée sur la simulation de l'ouvrage en remblai, dans un SIG, et d'une validation sur le terrain aboutit à des résultats proches. Les exemples présentés ici proviennent d'études réalisées ces dernières années sur les remblais des lignes à grande vitesse du TGV Est européen (dans le vignoble de Champagne) et du TGV Méditerranée (dans les terroirs arboricoles de la basse vallée de la Durance). Les résultats montrent des différences de températures de plusieurs degrés et aussi une stagnation plus longue de l'air froid superficiel en amont du remblai, ce qui peut provoquer la destruction des jeunes bourgeons. Devant l'importance des problèmes prévisibles, le Maître d'ouvrage a proposé des aménagements afin de réduire au mieux ce risque supplémentaire.

By radiativ weather (weak wind and clear sky), an embankment located in below slope level generates an obstacle with the natural flow of the cold air and it develops the formation of cold air lake. In spring, this obstacle can increase the frost risk for the vulnerable cultures (vine, fruit trees...). Weather measurements on fine scales and embankment simulation (with a plastic cover or in a GIS) permit to evaluate the temperature difference after the embankment implantation. The examples presented here are the impact of the embankments of high speed lines of the East European TGV (in the Champagne vineyard) and of the Mediterranean TGV (in orchards of Durance Valley). The results show a temperature difference of several degrees and also a longer stagnation of cold air surface upstream of the embankment, which can cause the buds destruction. With the importance of the foreseeable problems, the building owner proposed installations in order to reduce this additional risk.

\section{INDEX}

Mots-clés : mesures micrométéorologiques, ouvrage en remblai, risque climatique, viticulture, SIG

Keywords : arboriculture, micro-weather embankment, climate risk, vine growing, GIS 


\section{AUTEURS}

\section{HERVÉ QUENOL}

Hervé Quénol, herve.quenol@uhb.fr, est chargé de Recherche au CNRS. Ilfait partie du Laboratoire COSTEL, UMR6554 LETG, Université Rennes 2, Place du Recteur Henri Le Moal 35043 Rennes Cedex et il est en outre secrétaire de l'Association Internationale de Climatologie et secrétaire de la commission "SIG et Géomatique" du CNFG. Il a récemment publié :

- Quénol H., Bridier S., Frangi J.P., Beltrando G., de Rosny V.,2006.Space-time variability factors from air pollution inside Paris by CO measurements. Erdkunde, 60/1, 40-50.

- Quénol H., Monteiro A. et Beltrando G., 2007 : Evolution récente des terroirs viticoles dans la région do Entre Minho e Douro (nord du Portugal). Revue Internationale de Géomatique,(sous presse, $n^{\circ} 1$ ou $\left.2 / 2007\right)$.

\section{GÉRARD BELTRANDO}

UMR 8586 PRODIG, Université Denis Diderot

SÉBASTIEN BRIDIER

UMR 6012 ESPACE, Université de Provence 